\title{
Actions for Increasing the Credibility of Educational Research
}

\author{
Chair: Scott J. Peters \\ Discussant: Morgan S. Polikoff
}

\begin{abstract}
Words)
The purpose of education research is to better understand educational phenomena to inform policy and improve practice. Forward progress within any field is based on the validity and credibility of that field's research base - educators cannot make informed decisions based on anecdotal evidence, opaque research practices, or on studies that cannot be replicated.

Educational research is plagued by the same challenges as a number of fields - publication bias, barriers to research access, and seminal theories that have failed to replicate. This symposium will share four research practices that can increase the replicability and credibility of educational science. Each will be presented in terms of benefits and challenges for the researcher, the journal editor, and the educational knowledge base in general.
\end{abstract}

\section{Session Summary (500 Words)}

The advancement of science within any field is inherently progressive - any study builds on the existing literature. But what if those prior studies are not credible, not available to practitioners, or have never been subjected to independent replication? This creates a house of cards wherein practices are based on research that does not correctly explain the underlying phenomenon. For example, learning styles have long been popular in K-12 education despite virtually no evidence supporting the idea that individuals' instruction should be matched to their learning styles (Pashler, McDaniel, Rohrer, \& Bjork, 2009). The goal of this session is to introduce several mechanisms aimed to improve research quality and to assess their relevance and implementation viability in education.

Makel and Plucker (2014) reported that only 54\% of independent attempts at replication of published educational research were successful. Compounding the problems of false findings is a lack of systematic attempts at replication. Makel and Plucker further analyzed the entire publication histories of the top 100 education journals and found that only $0.13 \%$ attempted to replicate prior findings: 461 articles from 100 years of educational science. In any field, some number of published findings will be false. But without greater transparency and a culture of replication, false findings will continue to drive educational practice.

Despite a lack of systematic replications in the field, there reason for concern with the evidencebase, as the causes of low replicability are prevalent: publication bias in favor of "significant" findings, the ease with which an author can run multiple comparisons to achieve a publishable 
result, changing hypotheses after results are known (HARKing), and undisclosed flexibility of data reporting and analysis choices. These practices are harmful to the accuracy and credibility of science, yet are relatively common (John, Loewenstein, \& Prelect, 2012).

Concerns over false findings and failures to replicate seminal theories (e.g., growth mindset: Li $\&$ Bates, 2017) have lead other fields to implement Open Science practices. These practices include preregistering analysis plans prior to data collection, sharing data and research materials for evaluation and reuse, implementing registered reports as one form of academic publishing, posting preprints and providing open access to published research, and conducting large-scale collaborative research studies. Each of these methods has been shown to increase the validity of educational research and, thereby, better inform and support research-based practice in preK-12 and higher education (van der Zee, 2019).

This symposium will provide an overview of these four practices, explain why they can help increase the credibility and utility of educational research, respond to potential challenges with their implementation in education, and provide resources for how individuals can get started. The intended audience is both early career researchers as well as those who have little experience with open science practices, but who want to better understand what they look like in practice. The presenters were chosen because they are early implementers of each practice and are able to share their experiences both from the perspectives of researchers, faculty on promotion and tenure committees, journal editors, and reviewers.

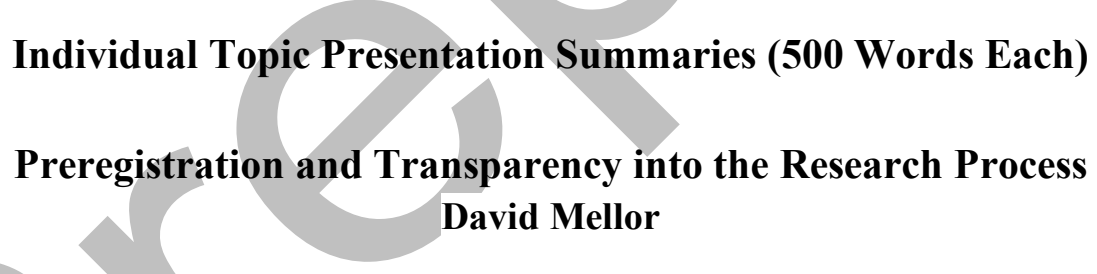

By submitting a detailed study and analysis plan to a registry prior to conducting a study, scientists can increase transparency into the research lifecycle while making a better distinction between planned, confirmatory hypothesis tests and unplanned, exploratory research. Both modes of research are important. However, without a clear distinction, one can unintentionally present results of exploratory analyses using statistical tools designed for a-priori hypothesis testing, which invalidates their diagnostic value. Furthermore, it presents results as being more surprising and publishable, at the expense of their credibility.

Preregistration is appropriate for hypothesis testing studies and for those where methodological decisions, such as variable construction or inclusion rules, can influence inferences. While some study designs present particular challenges for preregistration, such as longitudinal studies or those that rely on existing datasets, the benefits of transparency, better planning, and increased clarity between confirmation and discovery still adds value to the research. 
Additional measures to increase the evidential value of reported findings include data, code, and materials sharing, so that the complete research lifecycle can be better evaluated. This transparency increases the impact of reported findings, their credibility, and is particularly useful for original authors, who are better able to quickly adapt well curated materials for their own reuse.

Without preregistration, the scientific literature is prone to bias and exaggeration. The bias for statistically significant research findings is well known (Dwan, Gamble, Williamson, Kirkham, $\&$ the Reporting Bias Group, 2013), but it is also true that this bias produces a scientific literature that is unrepresentative of the total credible evidence. Franco, Malhotra, and Simonovits (2014) found that published research results were unrepresentative of the entire collected evidence even when null results were generated using common methodology. Preregistration can help researchers add credibility to their results, which are more likely to present credible null results (Kaplan \& Irvin, 2015) or more modest effect sizes than when preregistration is not used (Dechartres, et al., 2016; O’Boyle, Banks, \& Gonzalez-Mulé, 2017; Schäfer and Schwarz, 2019).

However, preregistration is no magic bullet. Preliminary evidence by Clasen and colleagues (2019) found that the first 27 preregistered studies published in Psychological Science deviated from their analysis plans without complete disclosure. This mirrors lessons learned from clinical trials, where outcome switching still occurs (Goldacre et al., 2019).

But even without complete adherence to preregistered plans, the act of preregistration increases transparency by making discoverable these shortcomings that would otherwise be impossible to detect. The onus is on the research community to gain experience with the process of preregistration and complete reporting. Finally, reviewers and authors must come to expect complete reporting of outcomes by not penalizing transparent changes to research designs. This shift in expectation must occur in tandem with increased expectation for preregistration when making inferential claims.

This session will introduce preregistration as a method as well as share some of the research cited above regarding impact. Resources for preregistering studies will be shared as will be suggestions for how to respond to common concerns or barriers.

\section{Registered Reports for Hypothesis-Driven Research Carly D. Robinson and Hunter Gehlbach}

\section{What are Registered Reports?}

Registered Reports are an approach to conducting and publishing empirical research. This process aspires to improve the transparency and reproducibility of hypothesis-driven research. In a Registered Report, authors first submit their Introduction, focal hypotheses, and Methods. This 
partial manuscript is sent out for peer review. At this point, a piece might receive a rejection, a revise and resubmit, or an "in principle acceptance." Once authors receive this in principle acceptance, the study is conducted. After the study's completion the full manuscript is submitted. Assuming no deviations from the plan outlined in the methods section and/or in the preregistration (Gehlbach \& Robinson, 2018), the paper is accepted. Thus, the acceptance or rejection of scholarly work is based on the quality of the question and methods rather than on the direction or effect size of the findings.

\section{Why Does Educational Research Need Registered Reports?}

For studies with a clear hypothesis, the Registered Report format has a number of benefits for researchers and the field more broadly. First, Registered Reports have been shown to reduce publication bias. In a 2019 study, Allen and Mehler found that across 113 published registered reports in the fields of biomedicine and psychology, 55\% failed to find support for their a priori hypotheses. This is far higher than the estimated $5 \%$ to $20 \%$ of traditional articles that result in null findings. Second, the format also appears to reduce questionable research practices by authors (Wiesman, Watt, \& Kornbrot, 2019). By requiring authors to register their methods and analysis plans in advance, there are fewer opportunities for research bias including $\mathrm{p}$-hacking and HARKing (the hindsight bias that is enacted by "hypothesizing after results are known") while still welcoming unregistered analyses that are clearly labelled as exploratory. Third, the Registered Report approach aligns editorial and author incentives towards publishing important questions with excellent research designs. Protocols are often accepted in advance of data being collected so it mitigates the risk of pursuing risky, novel projects (i.e., projects where the publishability of the outcome is contingent on the results, such as replication studies or resourceintensive projects) (Makel \& Plucker, 2014).

\section{Objectives \& Significance}

We explore the theoretical and practical implications for introducing Registered Reports as an accepted and common form of empirical work in education research. The specific objectives of this session are to:

1. Introduce the process for submitting a Registered Report in education;

2. Consider the advantages and disadvantages of this type of empirical research article for educational studies;

3. Provide lessons learned and best practices for submitting Registered Reports; and

4. Discuss implications for the field of education research, more broadly.

To make each of these four objectives more accessible to the audience, each will be discussed from the perspective of a reviewing editor as well as a submitting author. 
Establishing Registered Reports as a normal education research practice would result in the publication of a much greater number of null findings. In turn this would empower researchers and policymakers with a much better grasp on what works in education.

\section{Open Access and Preprints Amanda Montoya, Matthew C. Makel, Bryan Cook, and Kendal Smith}

\section{What are open access and preprints?}

Open access refers to content that is freely available and not behind a paywall. In applied fields like education, open access expands availability of research to the ultimate consumers: parents, practitioners, and policymakers. There are entire journals that are open access (e.g., AERA Open), whereas other journals have particular articles that are open access. The cost structure for open access can vary, but often includes author processing charges. Although this may feel like an additional burden to authors and something previously associated with publications lacking credibility, these fees are a cost shift from the consumer (e.g., institutional libraries) to the producer (e.g., researchers). The shift in cost is most relevant to authors, whereas the shift in accessibility is most relevant to consumers.

Preprints provide a complementary system to the traditional journal publication route. Preprints are unpublished versions of manuscripts. The first preprint server, arXiv, was created in 1991, now housing over 1.5 million papers. The Center for Open Science is home to many communitybased preprint services, including EdArxiv (launching August 2019). EdArXiv is a free, open source, non-profit service that allows education researchers to post their work. The term "preprint" can be misleading as the unformatted version of peer-reviewed manuscripts, often called postprints, can also be shared. Preprints have generally not yet been peer-reviewed. As such, preprints should be critically evaluated and their results taken as tentative.

\section{Value to Education Community}

Teachers and policymakers who cannot access research findings cannot base their actions on those findings. Moreover, researchers from developing countries often have severely limited access to the research base, and researchers from developed countries have incomplete access The traditional publishing model restricts access to information to a narrow group. Open access and preprints expand access to research to all.

In 2017, six AERA journals that are normally closed became open access for a two-month period. Gershenson, Polikoff, and Wang (2019) assessed the unadvertised openness and found that downloads in the newly-open journals increased by $60 \%-80 \%$. By increasing accessibility to research, more people read the research and, in theory, allowed for more educators to base their actions and instruction on the most-recent scientific knowledge. 
Preprints allow authors to get feedback prior to submission, disseminate their work, and avoid publication bias against null or unpopular results. Links to manuscripts on preprint servers remain static, making them useful for discoverability and researchers whose institutional affiliation changes. Moreover, although these results should be interpreted with caution, a recent analysis of preprints from bioRxiv found that manuscripts with a preprint received higher Altmetric scores and more citations than articles without a preprint (Serghiou \& Ioannidis, 2018).

\section{Resources \& Advice to Get Started}

Resources such as where to seek funds for author processing charges and preprint servers, how to determine which journals allow pre- and post-prints, and how to justify/explain the use of open access and preprint servers will be discussed. Lessons learned by those who have posted preprints and helped create EdArXiv will be shared.

\section{Large-Scale Collaborative Science: The ManyClasses Approach to Experimental Educational Research}

Emily R. Fyfe, Joshua R. de Leeuw, Paulo F. Carvalho, Robert L. Goldstone, Janelle Sherman, and Benjamin A. Motz

Complex questions require complex teams that leverage the expertise of professionals across a variety of disciplines (NRC, 2015). The science of education is no exception. To understand which instructional practices work for whom and under what conditions, projects need to expand beyond a single investigator and beyond a single educational context (https://ies.ed.gov/director/remarks/12-17-2018.asp).

The goal of ManyClasses is to provide a large-scale collaborative approach to education research in which we investigate the generalizability of educational practices in a wide range of contexts. One collaboration is among researchers with different areas of expertise. The core ManyClasses team includes six researchers with experience teaching various courses and with expertise in psychology, education, data science, and technology. Another collaboration is among researchers and teachers. One unique goal of a ManyClasses study is to maintain the rigor of a randomized experiment while also allowing teachers the flexibility to prepare materials that are authentic to their discipline. The first ManyClasses study will launch in Fall 2019 and will be represented in classes from at least 17 disciplines ranging from agriculture to business, online and in-person, and with enrollments ranging from 20 to 140 students. A third collaboration is among researchers and administrative officials. The first ManyClasses study will be at a minimum of four universities across eight campuses. The multi-institutional nature of the project requires precise attention to scientific integrity, student rights, and data security. As such, our current 
collaborations have included data stewards, chief information security officers, and associate general counsels.

There are at least three factors that make the current ManyClasses collaborations successful: transparency, communication, and perspective-taking. For large-scale education research to work, study protocols need to be clear to all parties involved. To that end, we created an osf webpage that documents the IRB protocol, the analysis plan, and instructor resources (https://osf.io/q84t7/). Further, the manuscript, which outlines the method and data collection details, was submitted as a registered report (Fyfe et al., 2019). Open communication is also a key priority. This includes biweekly meetings for the research team, a series of open webinars, calls for applications via twitter and listservs, and a central contact for instructors. Finally, perspective-taking has shaped the ManyClasses approach. Conducting an experiment in authentic courses has ramifications for many different people. For scientists, the experimental design increases the credibility of the findings. For teachers, changes to course materials influence course evaluations. For students, the target manipulation influences learning outcomes. For university personnel, sharing of student data influences legal and security issues.

In sum, the ManyClasses approach to education research is a unique large-scale collaboration that answers the calls for team science (e.g., NRC, 2015), open science (e.g., Peters, 2009), and systematic replications (https://ies.ed.gov/director/remarks/12-17-2018.asp). This session will share ideas for how researchers can engage in large-scale collaborative science, like ManyClasses, as well as present an argument for how such collaborations yield more-credible knowledge in the field of education.

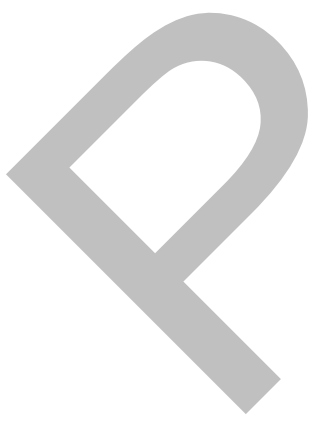




\section{References}

Allen, C., \& Mehler, D. (2019). Open science challenges, benefits and tips in early career and beyond. PLoS biology, 17(5), e3000246. doi:10.1371/journal.pbio.3000246

Claesen, A., Gomes, S. L. B. T., Tuerlinckx, F., \& vanpaemel, W. (2019, May 9).

Preregistration:

Comparing dream to reality. doi:10.31234/osf.io/d8wex

Dechartres, A., Ravaud, P., Atal, I., Riveros, C., \& Boutron, I. (2016). Association between trial registration and treatment effect estimates: A meta-epidemiological study. BMC Medicine, 14(1). doi:10.1186/s12916-016-0639-x

Dwan, K., Gamble, C., Williamson, P. R., Kirkham, J. J., \& the Reporting Bias Group. (2013). Systematic Review of the Empirical Evidence of Study Publication Bias and Outcome Reporting Bias - An Updated Review. PLoS ONE, 8(7), e66844. doi:10.1371/journal.pone.0066844

Franco, A., Malhotra, N., \& Simonovits, G. (2014). Publication bias in the social sciences: Unlocking the file drawer. Science, 345(6203), 1502-1505.

doi:10.1126/science. 1255484

Fyfe, E., de Leeuw, J. R., Carvalho, P. F., Goldstone, R., \& Motz, B. (2019).

ManyClasses 1: Assessing the generalizable effect of immediate versus delayed feedback across many college classes.https://doi.org/10.31234/osf.io/4mvyh

Gehlbach, H., \& Robinson, C. D. (2018). Mitigating illusory results through preregistration in education. Journal of Research on Educational Effectiveness, 11(2), 296-315. doi:10.1080/19345747.2017.1387950

Gershenson, S., Polikoff, M. S., and Wang, R. (2019). When paywals go AWOL: The demand for open access education research. Institute for Labor Economics Discussion Paper No. 12158. Retrieved from https://www.iza.org/publications/dp/12158/when-paywall-goesawol-the-demand-for-open-access-education-research

Goldacre, B., Drysdale, H., Dale, A., Milosevic, I., Slade, E., Hartley, P., ... Mahtani, K. R. (2019). COMPare: A prospective cohort study correcting and monitoring 58 misreported trials in real time. Trials, 20(1), 118. doi:10.1186/s13063-019-3173-2

John, L. K., Loewenstein, G., \& Prelec, D. (2012). Measuring the prevalence of questionable research practices with incentives for truth telling. Psychological Science, $23,524-532$.

doi:10.1177/0956797611430953

Kaplan, R. M., \& Irvin, V. L. (2015). Likelihood of Null Effects of Large NHLBI Clinical Trials Has Increased over Time. PLoS ONE, 10(8), e0132382. doi:10.1371/journal.pone.0132382

Li, Y., \& Bates, T. (2017). Does mindset affect children's ability, school achievement, or 
response to challenge? Three failures to replicate. Retrieved from https://osf.io/preprints/socarxiv/tsdwy/download?format=pdf

Makel, M. C., \& Plucker, J. A. (2014). Facts are more important than novelty: Replications in the education sciences. Educational Researcher, 43(6), 304-316. doi:10.3102/0013189X14545513

National Research Council (2015). Enhancing the effectiveness of team science. Washington, DC: The National Academies Press. doi:10.17226/19007

O’Boyle, E. H., Banks, G. C., \& Gonzalez-Mulé, E. (2017). The Chrysalis Effect: How Ugly Initial Results Metamorphosize Into Beautiful Articles. Journal of Management, 43(2), 376-399. doi:10.1177/0149206314527133

Pashler, H., McDaniel, M., Rohrer, D., \& Bjork, R. (2008). Learning Styles: Concepts and Evidence. Psychological Science in the Public Interest, 9(3), 105-119. doi:10.1111/j.1539-6053.2009.01038.x

Peters, M.A. (2009). Open education and the open science economy. Yearbook of the National Society for the Study of Education, 108, 203-225.

Schäfer, T., \& Schwarz, M. A. (2019). The meaningfulness of effect sizes in psychological research: Differences between sub-disciplines and the impact of potential biases. Frontiers in Psychology, 10, 1-13. doi:10.3389/fpsyg.2019.00813

Serghiou, S., \& Ioannidis, J. P. A. (2018). Altmetric scores, citations, and publication of studies posted as preprints. JAMA, 319(4), 402-404. doi:10.1001/jama.2017.21168

van der Zee, T., \& Reich, J. (2018). Open Education Science. AERA Open. doi: $10.1177 / 2332858418787466$

Wiseman, R., Watt, C., \& Kornbrot, D. (2019). Registered reports: An early example and analysis. PeerJ, 7, e6232. doi:10.7717/peerj.6232

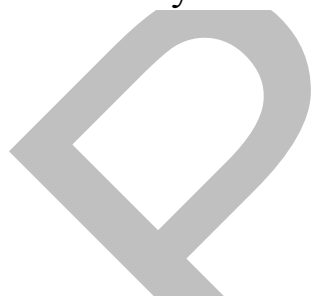

Authors listed alphabetically:

Carvalho, P. F., Cook, B., de Leeuw, J. R., Fyfe, E., Gehlbach, H., Goldstone, R. L., Makel, M., Mellor, D., Montoya, A., Motz, B. A., Peters, S. J., Polikoff, M. S., Robinson, C. D., Sherman, J., \& Smith, K. Actions for increasing the credibility of educational research. A symposium proposal submitted to the American Educational Research Association annual conference. San Francisco, CA. 